\title{
Determination of Vascular Contrast of Hemoglobin with Gold Nano-particles Using Near Infra Read
}

\author{
Parinaz Mehnati ${ }^{1}$, Bahman Alipour ${ }^{1,{ }^{*}}$, Roya Salehi ${ }^{2}$, Behroz Shabani ${ }^{3}$ \\ ${ }^{1}$ Medical Radiation Sciences Research Group, Department of Medical Physics, \\ Faculty of Medicine, Tabriz University of Medical Sciences, Tabriz, Iran \\ ${ }^{2}$ Department of Nano Medical, Faculty of Medicin, Tabriz University of Medical \\ Sciences, Tabriz, Iran \\ ${ }^{3}$ Department of Inorganic Chemistry, Faculty of Chemistry, Tabriz University, Tabriz, \\ Iran \\ * Corresponding author: Bahman Alipour, Medical Radiation Sciences Research \\ Group, Department of Medical Physics, Faculty of Medicine, Tabriz University of \\ Medical Sciences, Tabriz, Iran.E-mail:bahmanalipour69@gmail.com
}

DOI: $10.21859 /$ mci-supp-89

\section{Keywords:}

Vascular Contrast

Hemoglobin

Nano-particles

\begin{abstract}
Introduction: Early detection and treatment of breast cancer may be helping to save of the lives of patients. Many new techniques have been urban to detect breast cancer. One of them is the use of nano-particles for accuracy and early diagnosis after photo transfer. In this study, we used gold nanoparticles which can change light source transfer intensity. Material and Methods: Spherical gold nano particles and four types of bloods with $0,1,2,4$ hemoglobin concentrations used for simulating of breast cancerous conditions. Nanoparticles with each concentrations of hemoglobin injected into the breast phantom including vessels and transmitted light intensity measured by power meter. Light source was near infra red At 635 wavelength.

Results: The intensity of the passing lights from hemoglobin concentrations of $0,1,2$, and 4 were $9.31 \mathrm{mw}, 4.73 \mathrm{mw}, 3.36 \mathrm{mw}$ and $2.86 \mathrm{mw}$, respectively in without nano particle condition. When the nano-particles were blended with hemoglobin concentrations of 0 , 1,2 , and 4, the intensity of the passing light were $7.43 \mathrm{mw}, 3.62 \mathrm{mw}, 2.29 \mathrm{mw}$ and $2.02 \mathrm{mw}$, respectively.

Conclusions: This study showed that addition of Spherical gold nano-particles to different concentration of hemoglobin provides a effective decline on the light transferring intensity.
\end{abstract}

